\title{
Crash-Tolerant Causal Broadcast in $O(n)$ Messages
}

\author{
Achour Mostéfaoui $^{\circ}$ Matthieu Perrin $^{\circ}$ Michel Raynal $^{\diamond, \star}$, Jiannong Cao ${ }^{\star}$ \\ ${ }^{\circ}$ LS2N, Université de Nantes, 44322 Nantes, France \\ ${ }^{\diamond}$ Univ Rennes IRISA, Campus de Beaulieu, 35042 Rennes, France \\ ${ }^{\star}$ Department of Computing, Polytechnic University, Hong Kong
}

\begin{abstract}
Causal broadcast is a communication abstraction designed for asynchronous systems. It ensures that the messages broadcast by the processes are delivered in their broadcast causality order, namely, if the broadcast of a message $m$ causally precedes the broadcast of a message $m^{\prime}$, no process delivers $m^{\prime}$ unless it has previously delivered $m$. Several algorithms implementing causal broadcast have been proposed for asynchronous systems prone to any number of process crashes. These algorithms rely on an underlying Reliable Broadcast abstraction, whose message cost is $n^{2}$. This paper presents a simple causal broadcast algorithm whose cost is $n$ messages per causal broadcast. This is obtained at the cost of protocol messages whose size can be up to $n$ application messages. Hence, the proposed algorithm is particularly interesting for applications whose messages are small.
\end{abstract}

Keywords: Asynchronous system, Backward recursion, Broadcast abstraction, Causal broadcast, FIFO message delivery, Message-passing, Process crash failure. 


\section{Introduction: Computing Model and Causal Broadcast}

\subsection{Computing Model}

Process model The system is made up of a set of $n$ processes denoted $p_{1}, \ldots, p_{n}$. Each process is sequential (which means it executes one step at a time), and asynchronous (which means it progresses at its own speed, which can vary with time and remains always unknown to the other processes).

Any number of processes can crash. A crash is a premature halt (after it crashed, if it ever does, a process executes no more steps). A process that does not crash is called correct. A process that crashes is called faulty.

Communication model Processes can send and receive messages through channels. It is assumed that any pair of processes is connected by a bidirectional channel. Channels are reliable and asynchronous. Reliable means that messages are neither corrupted, nor duplicated, nor lost. Asynchronous means that, albeit finite, the transit duration of a message is arbitrary.

The sending and the reception of a message are atomic steps. The processes can also use a broadcast macro-operation, denoted broadcast $(m s g)$ where $m s g$ is a message, which is a shortcut for "for each $j \in\{1, \ldots n\}$ do send $(m s g)$ to $p_{j}$ end for". Let us observe that this macro-operation is not reliable, namely, if the sender crashes while executing it, an arbitrary subset of processes (possibly empty) receive the message.

A message created at the application level is called application message. A message generated by the algorithm implementing causal broadcast is called protocol message. When clear from the context, we use only the term message.

\subsection{Causal Broadcast}

Definition Causal broadcast was introduced by K. Birman and T. Joseph in a pioneering work on fault-tolerant distributed systems [3]. It is a communication abstraction that provides the processes with two operations denoted causal_broadcast() and causal_deliver(), that allow to broadcast and deliver messages. When a process invokes causal_broadcast $(m)$, we say it c-broadcasts the message $m$; similarly when the invocation of causal_deliver() returns the message $m$, we say it c-delivers the message $m$ (or $m$ is c-delivered). Without loss of generality, it is implicitly assumed that all messages c-broadcast by the processes are different (this can be easily realized by associating a pair - local sequence number, sender identity - with each message, as done in this article). Formally, causal broadcast is defined by the following properties.

- Validity. If a process c-delivers a message $m$ from a process $p_{i}, p_{i}$ previously c-broadcast $m$.

- Integrity. A process c-delivers a message $m$ at most once.

- Causal Delivery. If a process c-broadcasts a message $m$ and later c-broadcasts a message $m^{\prime}$, or a process c-delivers a message $m$ and later c-broadcasts a message $m^{\prime}$, no process c-delivers $m^{\prime}$ before c-delivering $m$.

- Termination. A message c-broadcast by a correct process is c-delivered by all correct processes.

Validity states that no spurious message can be c-delivered. Integrity states there is no message duplication. Causal Delivery states that the messages are c-delivered according to the causal links relating their c-broadcasts and c-deliveries [7]. Finally, Termination states that the correct processes c-deliver at least the messages they c-broadcast. 
Example A simple example of a causal broadcast execution is presented at the left of Fig. 1. Because they are c-broadcast by the same process $p_{1}$, no process can c-deliver $m_{1}$ before $m_{0}$. The same holds for $m_{3}$ and $m_{4}$ which are c-broadcast by $p_{2}$ : no process can c-deliver $m_{4}$ before $m_{3}$. As the c-broadcasts of $m_{2}$ and $m_{1}$ are not causally related, they can be c-delivered in a different order at distinct processes. The same holds for $m_{2}$ and $m_{3}$. Hence, the execution depicted in the figure satisfies the c-broadcast properties. Differently, if the message $m_{2}$ (i) had been delivered at $p_{2}$ before it issued the broadcast of $m_{3}$, and (ii) delivered before $m_{3}$ at process $p_{1}$, the execution would be incorrect. This is because $m_{3}$ would causally depend on $m_{2}$ and consequently no process would be allowed to deliver $m_{3}$ before $m_{2}$.

Figure 1: A causal broadcast execution and its message precedence graph

A short historical perspective As already said, causal broadcast was introduced by K. Birman and T. Joseph in [3]. Its first implementation was done in the ISIS system [2], where each message was required to carry its full causal past. A more efficient implementation was then introduced [4]. While it is now well accepted as a meaningful communication abstraction, causal broadcast initiated a controversy in the operating system community (see e.g., [1]). Several causal broadcast algorithms have been presented in the literature (e.g., see the textbooks [5, 11]), which use an underlying reliable broadcast abstraction.

Efficient implementations of causal broadcast for failure-free systems are presented in several articles and textbooks (e.g., $[10,12,13,14])$. Formal characterizations of causal broadcast can be found in $[6,8,10]$.

A more low-level approach was introduced in [9], where is defined the notion of a conversation, close to causal broadcast. The presentation of this paper is operating system-oriented and no precise definitions (i.e., properties-based) are given.

Motivation All the causal broadcast algorithms suited to crash-prone asynchronous message-passing systems we know are based on an underlying reliable broadcast abstraction. To ensure that each application message c-delivered by a correct process is c-delivered by all correct processes, all reliable broadcast algorithms use $O\left(n^{2}\right)$ protocol messages for each application message. However, while it is sufficient, it has never been proved that reliable broadcast was necessary to implement causal broadcast. Hence our investigation on the implementation of causal broadcast, which has given rise to the simple algorithm presented in this paper. This algorithm requires $O(n)$ protocol messages for the c-broadcast of an application message. This is obtained at the price of protocol messages sometimes carrying a few application messages (this depends on the application communication pattern). Hence, the proposed algorithm seems suited to the case where application messages are small. The principle that underlies the algorithm is the use of a precedence graph, which captures the causality relation on application messages. In some sense, the proposed algorithm can be seen as a variant of a reliable broadcast algorithm customized to causal broadcast.

Precedence graph Such a graph $G$ is associated with each execution. Its vertices are the application messages. As far as its edges are concerned, there is a directed edge from a message $m$ to a message $m^{\prime}$ if (i) the invocations of causal_broadcast $(m)$ and causal_broadcast $\left(m^{\prime}\right)$ are consecutive invocations by the same process $p_{i}$, or (ii) the c-delivery of $m$ and the invocation of causal_broadcast $\left(m^{\prime}\right)$ by a process $p_{j}$ are consecutive in the sense there is no invocation of causal_broadcast () by $p_{j}$ between them. The figure depicts the precedence graph of the execution on its right. If the directed edge $\left(m, m^{\prime}\right)$ belongs to $G$, we say that $m^{\prime}$ causally depends (directly) on $m$, or $m$ is an immediate causal predecessor of $m^{\prime}$. If there is a directed path from $m$ to $m^{\prime}$, we say that $m^{\prime}$ depends (transitively) from $m$, or $m$ is a causal predecessor of $m$. Two messages $m$ and $m^{\prime}$ that are not causally related are said to be independent. The 
causal past of a message $m$ is the set of all the messages $m^{\prime}$ such that there is a directed path from $m^{\prime}$ to $m$.

\section{An Efficient Causal Broadcast Algorithm}

Algorithm 1, described below, implements causal broadcast. The messages that are c-broadcast are called application messages, while the messages generated by the algorithm are called protocol messages. As already indicated, this algorithm ensures that each invocation of causal_broadcast () generates $n$ protocol messages.

Local variables at a process $p_{i} \quad$ Each process manages two local variables.

- $s n_{i}$ is an integer variable, initialized to 0 , used to associate a sequence number with each message c-broadcast by $p_{i}$.

- copr $_{i}$ (for compressed predecessors is a sequence of triplets $\langle m, j, s n\rangle$ such that the message $m$ has been c-broadcast by process $p_{j}$ with sequence number $s n$. A triplet $\langle m, j, s n\rangle$ is contained into $\operatorname{copr}_{i}$ if $p_{i}$ c-delivered $m$ after its last c-broadcast and did not c-deliver any more recent message from $p_{j}$ later (see Fig. 2). ${ }^{1}$

The symbol $\epsilon$ denotes the empty sequence, and $\oplus$ denotes the concatenation of a new element at the end of the sequence.

Figure 2: Meaning of the sequence copr $r_{i}$

Considering a process $p_{i}$, the meaning of $c o p r_{i}$ is illustrated on Fig. 2, in which $p_{i}$ invoked first causal_broadcast $\left(m_{1}\right)$ and later causal_broadcast $\left(m_{2}\right)$. Moreover, between these two consecutive invocations, $p_{i}$ c-delivered the messages $m, m^{\prime}$, and $m^{\prime \prime}$ (in this order). This means that, to ensure a correct message c-delivery order, (i) no process must c-deliver $m_{2}$ (i) before $m_{1}$ (because $m_{2}$ was c-broadcast later by the same process $p_{i}$ ), and (ii) before the messages $m, m^{\prime}$, and $m^{\prime \prime}$ (because their c-deliveries at $p_{i}$ causally precede the c-broadcast of $m_{2}$ ). Hence, copr $_{i}$ is used to register all the messages c-delivered by $p_{i}$ between any two consecutive invocations of causal_broadcast () it issues.

On the client side: the operation causal_broadcast $(m)$ The code of this operation is pretty simple. Process $p_{i}$ first increases $s n_{i}$, and then broadcasts the message seqmsg, which contains the sequence $\operatorname{copr}_{i} \oplus\left\langle m, i, s n_{i}\right\rangle$ (lines 1-3). Let us notice that the triplet associated with the last message previously c-broadcast by $p_{i}$ is suppressed from copr $r_{i}$ before the broadcast of seqmsg. As $p_{i}$ did not crash during the previous broadcast, this message is received by all processes, and the only ordering information needed is its sequence number, namely $\left(s n_{i}-1\right)$, which is explicitly encoded in the triplet $\left\langle m, i, s n_{i}\right\rangle$.

Let us observe that any protocol message seqmsg contains at least one triplet. The sequence of triplets $\operatorname{copr}_{i}$ will be used as a causal barrier imposing to c-deliver all its messages before $m$. As just indicated, the sequence number $s n_{i}$ will be used to ensure that the previous message c-broadcast by $p_{i}$ is c-delivered before $m$.

\footnotetext{
${ }^{1}$ The timstamps associated with messages of copr $_{i}$ are the one that compose compressed vector clocks in [2]. Let us observe that the compressed predecessors of $p_{i}$ include at most one messages from $p_{j}$ if $p_{i}$, but can contain none of them. Said differently, $\left|\operatorname{copr}_{i}\right| \leq n$.
} 


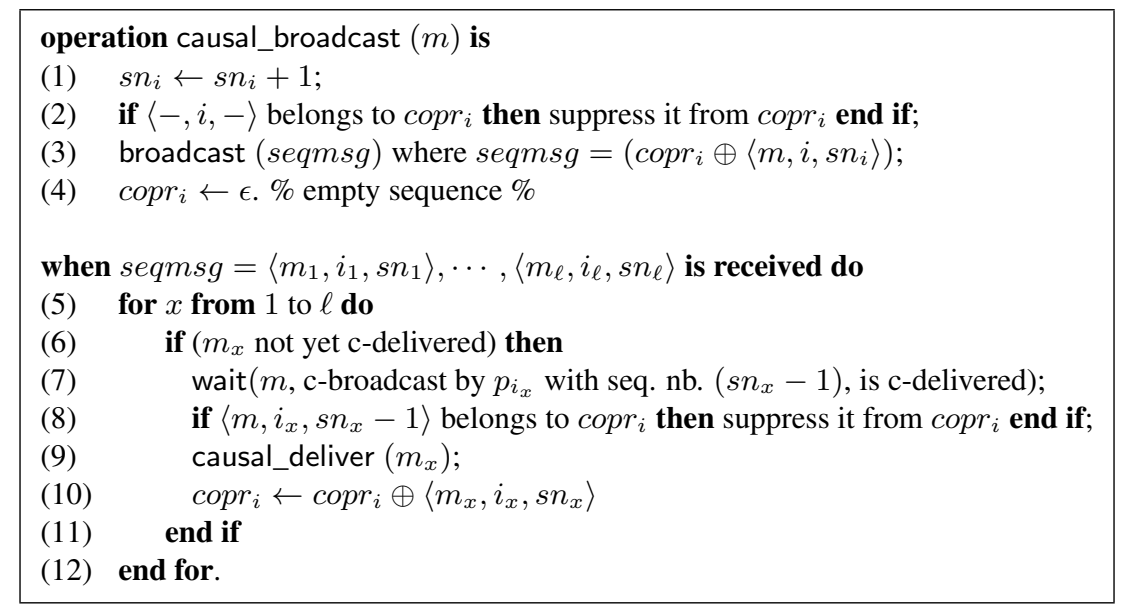

Algorithm 1: Efficient causal broadcast (code for $p_{i}$ )

On the server side: Message reception When a process $p_{i}$ receives a protocol message seqmsg containing a sequence $\left\langle m_{1}, i_{1}, s n_{1}\right\rangle, \cdots,\left\langle m_{\ell}, i_{\ell}, s n_{\ell}\right\rangle$, it considers each of its triplet, one after the other ( "for" loop, lines 5-12). Let $\left\langle m_{x}, i_{x}, s n_{x}\right\rangle$ the current triplet. It means that $m_{x}$ was c-broadcast by $p_{i_{x}}$, and its sequence number is $s n_{x}$. There are two cases.

- If $m_{x}$ has already been locally c-delivered, $p_{i}$ proceeds to the next triplet, if any.

- If $m_{x}$ has not yet been locally c-delivered, $p_{i}$ first waits until the previous message (say $m$ ) cbroadcast by $p_{i_{x}}$ is c-delivered (we can assume that each protocol message reception generates a new thread in charge of the triplets in seqmsg). This is made possible thanks to the sequence number $s n_{x}$ (line 7). After this message $m$ has been c-delivered, it is suppressed from copr $_{i}$ (line 8). This is due to the fact that the causal dependence between this message $m$ and the next message c-broadcast by $p_{i}$ must be updated, namely replaced by the new causal dependence between $m_{x}$ and the next message c-broadcast by $p_{i}$ (line 8 ). Finally, $m_{x}$ can be c-delivered (line 10).

As we can see, each iteration step of the "for" loop (lines 5-12) has a backward recursive flavor, captured by the "wait" statement at line 7 . This statement entails a recursive messages delivery, whose aim is to guarantee causal delivery.

\section{Proof of the Algorithm}

Lemma 1 Each local variable copr ${ }_{i}$ contains at most $n$ triplets.

Proof A local sequence $\operatorname{copr}_{i}$ is initialized to the empty sequence $\epsilon$, and reset to this value each time $p_{i}$ invokes causal_broadcast(). Then, when $p_{i}$ adds a new triplet $\left\langle m_{x}, i_{x}, s n_{x}\right\rangle$ to $\operatorname{copr}_{i}$ (line 10), it previously suppresses the triplet $\left\langle-, i_{x}, s n_{x}-1\right\rangle$ if it is in $\operatorname{copr}_{i}$ (line 8). Hence, for any $i_{x}$, copr $_{i}$ contains at most one triplet $\left\langle-, i_{x},-\right\rangle$, which proves the lemma.

The next corollary is an immediate consequence of the previous lemma and lines 2-3.

Corollary 1 A protocol message contains at most $n$ triplets.

Lemma 2 Let us consider any two consecutive application messages $m$ and $m^{\prime} c$-broadcast by a process $p_{j}$. No process $p_{i}$ c-delivers $m^{\prime}$ before $m$. 
Proof The proof is an immediate consequence of the "wait" statement of line 7, which imposes a FIFO delivery order between each pair of processes.

Lemma 3 Let us consider any two application messages $m$ and $m^{\prime}$ such that causal_broadcast $(m)$ causally precedes causal_broadcast $\left(m^{\prime}\right)$. No process $c$-delivers $m^{\prime}$ before $m$.

Proof If $m$ and $m^{\prime}$ are c-broadcast by the same process, the proof follows from Lemma 2. Let us now consider the case where $m$ and $m^{\prime}$ are c-broadcast by different processes $p_{i}$ and $p_{j}$. Let $s n$ be the sequence number associated with $m$. As causal_broadcast $(m)$ causally precedes causal_broadcast $\left(m^{\prime}\right)$, there is a directed path of messages in the precedence graph $m=m_{1}, m_{2}, \ldots, m_{k}=m^{\prime}$, where $k \leq n-1$, and an associated sequence of distinct processes $p_{j(1)}=p_{i}, p_{j(2)}, \ldots, p_{j(k)}=p_{j}$ such that

- $m=m_{1}$ has been c-broadcast by $p_{j(1)}=p_{i}$ and c-delivered by $p_{j(2)}$ (in fifo order),

- after $p_{j(2)}$ c-delivered $m_{1}$, it c-broadcast $m_{2}$, which was c-delivered by $p_{j(3)}$ (in fifo order), etc.,

- after $p_{j(k)}=p_{j}$ c-delivered $m_{k-1}$ (in fifo order), it c-broadcast $m^{\prime}=m_{k}$.

Due to lines 7 and 9-10, and the definition of seqmsg at line 3, it follows that, for any $x \in[1 . . k]$, $c o p r_{j(x)}$ includes, for each process $p_{y}$, the last causal predecessor of $m_{x}$ c-broadcast by $p_{y}$ (if any). Hence, when a process receives seqmsg $=\langle-,-,-\rangle, \cdots,\left\langle m^{\prime}, j,-\right\rangle$, the triplets in seqmsg capture the messages that must be c-delivered by $p_{j}$ before $m^{\prime}$, and one of these triplets is equal to $\left\langle-, i, s n^{\prime \prime}\right\rangle$ where $s n^{\prime \prime} \geq s n$. It follows that $p_{j}$ cannot c-deliver $m^{\prime}$ before $m$.

$\square$ Lemma 3

Remark The previous lemma shows that, from a "basic principles" point of view, Algorithm 1 is a reduction of message causal delivery to (process-to-process) FIFO message deliveries.

Lemma 4 If a correct process (i) c-broadcasts a message $m$, or (ii) $c$-delivers a message $m$ and later $c$-broadcasts a message $m^{\prime}$, the message $m$ is $c$-delivered by all correct processes.

Proof Proof of (i). Let us assume by contradiction that there are application messages c-broadcast by correct processes that are never c-delivered by some correct process. Let $m$ be a message and $p_{j}$ its correct sender, such that $m$ is the first message that is not c-delivered by a correct process $p_{i}$, while $p_{i}$ c-delivers all its causal predecessor messages. This permanent blocking occurs at line 7 . As $p_{j}$ is correct, it broadcast a protocol message seqmsg $=(\operatorname{copr} \oplus\langle m, j, s n\rangle)$. As $m$ is the "first" (as defined above) message entailing the permanent blocking of $p_{i}$, all the messages in the prefix sequence copr, are c-delivered ${ }^{2}$. Consequently, the processing of all triplets in copr terminates, and $p_{i}$ processes then $\langle m, j, s n\rangle)$ (last iteration of the "for" loop). When this occurs, $p_{i}$ remains blocked forever waiting for the previous message (say $m^{\prime}$ ) c-broadcast by $p_{j}$. But this permanent blocking means that $m$ is not the first message for which $p_{i}$ waits forever. This contradicts the assumption on $m$, and consequently, there is no "first" message from $p_{j}$ never c-delivered by $p_{i}$.

Proof of (ii). If a process $p_{i}$ c-delivers a message $m$ from a (correct or crashed process) $p_{j}$, it adds the triplet $\langle m, k, s n\rangle$ (where $p_{k}$ is the sender of $m$, and $s n$ its sequence number) to $c o p r_{i}$ at line 10. Hence, this triplet will be broadcast when $p_{i}$ will issue it next invocation of causal_broadcast(). $\quad \square_{\text {Lemma } 4}$

Theorem 1 Algorithm 1 implements the Causal Broadcast abstraction. Moreover, an invocation of causal_broadcast () costs $n$ protocol messages, and each protocol message carries at most $n$ application messages.

\footnotetext{
${ }^{2}$ Let us observe that it is possible that the message seqmsg broadcast by $p_{j}$ carries a triplet $\left\langle m^{\prime \prime}, k,-\right\rangle$ such that $p_{k}$ crashed while broadcasting the sequence of triplets $\langle-,-,-\rangle, \cdots,\left\langle m^{\prime \prime}, k,-\right\rangle$, and this sequence was never received by $p_{i}$. But in this case, if any, $p_{k}$ did not crash during its previous invocation of broadcast () and consequently, $p_{i}$ eventually receives the last message c-broadcast by $p_{k}$ before $m^{\prime \prime}$.
} 
Proof The Validity property follows directly from the text of the algorithm and the reliability of the network (none of them creates messages nor modifies their content). The Integrity property follows from line 6. The Causal Delivery property follows from Lemma 3 and the Termination property follows from part (i) of Lemma 4. The message cost properties follows from Lemma 1 and Corollary $1 . \square_{\text {Theorem }} 1$

\section{Ensuring a Stronger Termination Property}

A stronger termination for crashes during an invocation of broadcast ()$\quad$ As already indicated, if a process crashes while executing broadcast $(s e q m s g)$, some processes can receive seqmsg, while others do not. Let $P 1$ be the former set of processes, and $P 2$ the later. If the correct processes of $P 1$ (if any) do not issue new invocations of causal_broadcast() (whose internal broadcast () will forward messages in seqmsg to the processes of $P 2$ ), the correct processes of $P 2$ would never c-deliver messages in seqmsg sent by processes that crashed during their last invocation of broadcast(). This raises the following question. In addition to the basic termination property, how to ensure the following stronger termination property:

- Strong Termination. If a correct process $p_{i}$ c-delivers a message $m$, the message $m$ is c-delivered by all correct processes.

This Strong Termination property ensures that all correct processes c-deliver the same set of messages. If the message $m$ has been c-broadcast by a correct process, the basic termination property ensures strong termination. So, we have only to consider the case where the sender of $m$ crashed while it was executing the unreliable macro-operation broadcast(). Hence, answering the question amounts to ensure that any message that (i) is broadcast by a process that crashes and (ii) is c-delivered by a correct process, is c-delivered by all correct processes.

A look at Lemma 4 To this end, we rely on part (ii) of Lemma 4. This lemma states more than the termination property, which demands only that, if a correct process c-broadcasts a message $m$, all correct processes c-deliver $m$. It additionally states that, if a correct process c-delivers a message $m$ and later c-broadcasts a message $m^{\prime}$, the message $m$ is c-delivered by all correct processes. In particular, if all correct processes repeatedly c-broadcast messages during an infinite execution, then the algorithm ensures strong termination.

From termination to strong termination It follows from the previous discussion that the strong termination property can be obtained as follows. Let $p_{i}$ be a process that after some time does no longer invoke causal_broadcast(). This process is then required to c-broadcast a control application message (encoded in a default value, e.g., $\perp$ ), only if its variable copr $_{i}$ contains non- $\perp$ application messages. According to the algorithm, this invocation entails the required forwarding of the compressed predecessor application messages (which always succeeds if $p_{i}$ is not a faulty process). ${ }^{3}$

A note on complexity For each message $m$ c-broadcast by a process $p_{i}$, at most one message $\perp$ might be c-broadcast by any other process $p_{j}$. Therefore, in the worst case, this strategy might lead to the same number of messages as causal broadcast algorithms based on reliable broadcast. However, in practice, there are common situations in which the complexity would be much lower: (1) processes that

\footnotetext{
${ }^{3} \mathrm{~A}$ similar issue occurs when one has to solve early-deciding consensus in crash-prone synchronous message-passing systems. When a process attains a round $r$ in which it knows the decision estimates of all the processes that were not crashed at the beginning of round $r$, it can compute the decision value and decide it. But, as it has no means to know if the other processes know the same set of estimate values, it must execute an additional round during which it propagates the decision value before terminating (see [11] for more details).
} 
repeatedly c-broadcast messages never need to c-broadcast $\perp$ messages, and (2) one $\perp$ message can be used to forward up to $n-1$ messages. In particular, in an execution where processes first jointly cbroadcast and c-deliver $x \geq n$ messages, and only then c-broadcast one $\perp$ message each, $O(n x)$ protocol messages will be exchanged between processes, compared to $O\left(n^{2} x\right)$ messages for an algorithm based on reliable broadcast.

\section{Conclusion}

This article has presented a causal broadcast algorithm for asynchronous message-passing systems in which any number of processes can crash. Differently from causal broadcast algorithms based on reliable broadcast (which requires $O\left(n^{2}\right)$ protocol messages for each causal broadcast), the proposed algorithm generates only $n$ protocol messages per causal broadcast. To this end, it replaces "early message forwarding" used in reliable broadcast by "late message forwarding", and reduces causal message delivery to fifo message delivery.

\section{Acknowledgments}

This work was partially supported by the French ANR project 16-CE40-0023-03 DESCARTES devoted to layered and modular structures in distributed computing. The authors want to acknowledge the referees for their constructive comments.

\section{References}

[1] Birman K.P., A response to Cheriton and Skeen's criticism of causal and totally ordered communication. Operating Systems Review, 28(1):11-21 (1994)

[2] Birman K.P. and Cooper R., The ISIS project: real experience with a fault tolerant programming system. Proc. 4th ACM SIGOPS European workshop, Operating Systems Review, ACM Press, 25(2):103-107 (1991)

[3] Birman K.P. and Joseph T.A., Reliable communication in the presence of failures. ACM Transactions on Computer Systems, 5(1):47-76 (1987)

[4] Birman K.P., Schiper A., and Stephenson P., Lightweigt causal and atomic group multicast. ACM Transactions on Computer Systems, 9(3): 272-314 (1991)

[5] Cachin Ch., Guerraoui R., and Rodrigues L., Reliable and secure distributed programming, Springer, 367 pages, ISBN 978-3-642-15259-7 (2011)

[6] Kshemkalyani A. and Singhal M., Necessary and sufficient conditions on information for causal message ordering and their optimal implementation. Distributed Computing, 11(2):91-111 (1998)

[7] Lamport L., Time, clocks, and the ordering of events in a distributed system. Communications of the ACM, 21(7)-558-565 (1978)

[8] Murty V.V. and Garg V.K., Characterization of message ordering specifications and protocols. Proc. 7th Int'l Conference on Distributed Computer Systems (ICDCS'97), IEEE Press, pp. 492-499 (1997)

[9] Peterson L.L., Bucholz N.C., and Schilchting R.D., Preserving and using context information in interprocess communication. ACM Transactions on Computer Systems, 7(3):217-246 (1989)

[10] Raynal M., Distributed algorithms for message-passing systems. Springer, 510 pages, ISBN 978-3-64238122-5 (2013) 
[11] Raynal M., Fault-tolerant message-passing distributed systems: an algorithmic approach. Springer, 550 pages, ISBN: 978-3-319-94140-0 (2018)

[12] Raynal M., Schiper A., and Toueg S., The causal ordering abstraction and a simple way to simple to implement it. Information Processing Letters, 39(6):343-350 (1991)

[13] Schiper A., Eggli J., and Sandoz A., A new algorithm to implement causal ordering. Proc. 3rd Int'l Workshop on Distributed Algorithms (WDAG'89), Springer LNCS 392, pp. 219-232 (1989)

[14] Schwarz R. and Mattern F., Detecting causal relationships in distributed computations: in search of the Holy Grail. Distributed Computing, 7:149-174 (1994) 